\title{
SCIENCE
}

NEW YORK, MARCH 31, 1893.

ON THE ETHNOLOGICAL CHARACTERISTICS OF THE HUMAN NASAL CANALS, CONSIDERED AS AN ECONOMIC ADAPTATION.

BY WILlIAM C. BRAISLIN, M.D, BROOKLYN, N.Y.

THE human voice bears a constant relation to the physical construction of the voice-producing organs.

Deep, manly tones are the constant sequelæ to a larynx of large proportions, to long and slowly vibrating vocal cords; while the childish treble and the high-pitched voice of the female are the natural productions of the smaller larynx with its shorter, and, consequently, more rapidly vibrating, vocal cords.

Of equal importance in the modulation, tone, and indefinable individual peculiarity of the voice, is the construction of the nose and pharynx. The anatomical construction of these organs is as varying as is that of the facial features, and, to the trained eye, the individual peculiarities are as apparent.

It is not to be wondered at, therefore, that the racial peculiarities, in regard to the anatomical structures, of the internal nares are particularly striking.

The external anatomical construction of the nasal organ characteristic of the native African, is as distinguishing an ethnological differentiation as are the color of his skin, the texture of his hair, and the development of his brain.

Corresponding distinctions characterize the internal structure of this portion of the upper air tract of this race. Immediately on passing the anterior openings of the nares, the human nasal canals spread out into deeper and wider channels. These cavities. separated by the septum narium, are corrugated on their opposite surfaces by the three pairs of turbinated bodies, whose "curledleaf" surfaces increase enormously the extent of surface area of the nasal mucous membrane.

The bones of the cranium taking part in the formation of the nasal cavities, so differ in the African race as to make these canals wider, shorter, and less deep than those of other races. The bony ridges coursing their long axes, known as the turbinated bones, are also more blunt and less prominent and have less of the "curled-leaf" character.

Measurements of a single specimen of an a verage skull of either race will suffice to show this difference in bony framework.

\begin{tabular}{|c|c|c|}
\hline & Negro. & White. \\
\hline & $\begin{array}{l}\text { Centi- } \\
\text { metres. }\end{array}$ & $\begin{array}{l}\text { Centi- } \\
\text { metres. }\end{array}$ \\
\hline Width of anterior nasal canals.......................... & 2.7 & 2.5 \\
\hline Height of anterior nasal canals............................. & 2.6 & 3.5 \\
\hline $\begin{array}{l}\text { Length of nasal canals (from anterior nasal spine to pos- } \\
\text { terior nasal spine }) \ldots \ldots \ldots \ldots \ldots \ldots \ldots \ldots \ldots \ldots \ldots \ldots\end{array}$ & 52 & 5.3 \\
\hline $\begin{array}{l}\text { Length of nasal canals (from anterior nasal spine to pos- } \\
\text { terior-superior angle of vomer) } \ldots \ldots \ldots \ldots \ldots \ldots \ldots\end{array}$ & 7.0 & 7.2 \\
\hline 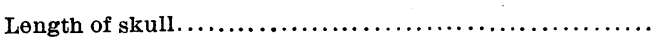 & 19.0 & 19.0 \\
\hline Breadth of skull $\ldots \ldots \ldots \ldots \ldots \ldots \ldots \ldots \ldots \ldots \ldots \ldots \ldots \ldots$ & 14.0 & 14.0 \\
\hline
\end{tabular}

The continuance of these peculiarities in the lineal descendants of the negro race as seen in America to-day, is markedly striking to the student of the comparative anatomy of this region.

One, indeed, cannot fail to note in any of the clinics devoted to the diseases of this region the increased advantage which accrues to the possessor of the wider nasal canals when pathological conditions, resulting in thickening of the lining mucous membrane, attack this portion of the anatomy.

One of the most prominent American larynxologists was enabled, by means of the more patent condition of the nasal canals in his negro patients, ${ }^{1}$ - the American descendants of this race,to make a contribution of certain facts to medical science, not otherwise easily obtainable.

Mr. Wallace ${ }^{2}$ supposes that the origin of the African race was due to a migration of some part of the race of ancestral man from the great Euro-Asiatic plateau, and that his present characteristics are the result of phssiological modifications due to climatic influence.

If we accept this likely explanation in regard to the special anatomical construction of the nasal cavities of the negro and his descendants, our logical conclusion is that they must present characteristics specially adapted for preparing the inspired air of a tropical climate for reception into the lung structures. We must suppose that ages of contact of his nasal mucous membrane with the atmosphere of a tropical climate have brought about, by the laws of natural selection, a nasal construction the most nearly adapted to dealing with the problem of the irritating qualities of a tropical atmosphere, of any existing race.

It does not seem probable that the evolution of the construction of the nasal canals, characteristic of the African type, has been due to any reason of better serving the purposes of the gustatory sense. The custom of regarding the nose as the organ of smell has, very properly, become modified to that of regarding it as primarily an organ of respiration. It is the proper channel for the conduction of air to the lungs for purposes of oxidation.

As has been stated, the means of warming, of moistening, and of freeing the air from dust and other irritating qualities are here most admirably afforded. The sense of smell must, indeed, also be regarded as a protective provision for the avoidance of substances irritating to the more delicate lung substance.

Had its purposes demanded an assistance to the procuring of ford or to the avoidance of poisonous food, a development of the gustatory sense, such as is found in the dog or other animals, would have been found in the human species. This, however, is not the case. The acute sense of smell is not one highly developed or inherent in man. Indeed, this sense is soon entirely lost in conditions of the nasal canals which interfere with its respiratory function.

The difference we have noted in the anatomical construction of this portion of the upper air tract - the nasal canals - is naturally resultant in different pathological effects in the different races as regards the particular portions of the respiratory tract most frequently becoming the seat of disease processes under the influence of atmospheric irritation. Less protection is afforded the lung structures of the negro race on account of the anatomical structure of his nasal canals, since less opposition to the irritating factors of the atmosphere is presented, by reason of the more patent and more direct course which the inspired air encounters. That the lung structure of this race in the United States suffers from the irritation caused by degenerating atmospheric conditions seems to the writer to be evinced by data of the Tenth Report of Vital Statistics of the United States, compiled by Dr. John S. Billings.

Among many other facts" of great significance in this compilation we are shown that the number of deaths per 1,000 from consumption - the disease most dependent, perhaps, upon irritating qualities of the atmosphere - is not only greater among the colored portion of our population, but it is in direct proportion

\footnotetext{
1 American Journal of the Medical Sciences, 1883.
}

2 "Darwinism," p. 460. Macmillan. 
greater the more severe the rigors of climate encountered in the respective areas of territory from which statistical returns are cited. Thus in the Gulf coast region the proportion of deaths from consumption per one thousand deatbs is about equal among the whites and blacks; but in the Middle Atlantic coast region the difference of numbers shown is a very distinct one.

In five specified areas of territory the exact proportion is as follows :-

The Number of Deaths from Consumption in 1,000 Deaths from all Causes.

\begin{tabular}{|c|c|c|}
\hline & Whites. & Colored. \\
\hline Middle A tlantic Coast Region....... & 140.9 & 175.1 \\
\hline South Atlantic Coast Region....................... & 880 & 105.5 \\
\hline Gulf Coast Region $\ldots \ldots \ldots \ldots \ldots \ldots \ldots \ldots \ldots \ldots \ldots \ldots \ldots \ldots$ & 115.8 & 120.6 \\
\hline The Interior Plateau..... & 138.4 & 126.8 \\
\hline The Ohio River Belt........ & 150.7 & 2381 \\
\hline
\end{tabular}

Thus, while in the first case there is a difference of 34.2 , in the third - the Gulf coast region - there is only a difference of a little less than 5 ; while in the fifth case a difference of 87.4 in the number of deaths per 1,000 from consumption exists.

We have, therefore, it seems to the writer, sufficient grounds upon which to advance the theory that the more patent condition of the nasal canals in the colored race is largely responsible for the more frequent occurrence in this race of lung disorders, as compared with the white races, in the United States.

Diseased conditions of the nasal canals in the dark race resulting in stenosis are especially rare; while, as every physician engaged in the clinical study of these disorders will testify, a condition of stenosis is one of the most common, and among the first, symptoms of disease involving this portion of the air tract in individuals of European descent.

It is unnecessary to say that the inference must not be drawn that a condition of stenosis is a safeguard against lung disorders. Any condition of the nasal passages which results in or necessitates mouth-breathing directly favors diseases of the lungs.

When a condition of the nasal passages exists prohibiting the free passage of air through them, the professional services of a physician or surgeon should be sought to remedy this defect. And since, as has been said, the nasal passages should be considered an integral part of the respiratory tract, the remedy should constitute not merely the rendering of this organ patent, but should also aim to restore it to a condition in which it may regain the ability of performing its normal functions of moistening, of warming, and of purifying, by freeing from irritating factors, the inspired air.

It is impossible to state how far the remedial effects of selection or evolution are modifying the peculiarities of anatomical structure of the nasal passages of the black race in the United States.

That these peculiarities become less prominent along with other racial peculiarities of the descendants of the negro race is, however, very evident to the accurate observer. To the untrained eye, the external characteristics of this organ are undergoing modification, and to clinical observers a like change is noted in the internal structure; but just how far this may be due to the admixture of white blood, and how far to selective and developmental modification, is beyond the power of the writer to estimate.

Professor W. S. Bayley of Colby University, Waterville, Me., has collected into a volume, with a separate title-page and index, notes on mineralogy which have appeared during 1892 in the American Naturalist. Professor Bayley is the editor of the Department of Mineralogy and Petrography of the Naturalist, and these notes summarize the papers that have been published during the past year. The volume will prove useful to those who wish to be posted on the literature of these two branches of science.

\section{THE EFFECT ON THE COLLEGE CURRICULUM OF THE INTRODUCTION OF THE NATURAL SCIENCES.}

\author{
BY W. L. POTEAT, WAKe forest COlLege, N.c.
}

THE natural sciences are at last firmly lodged in the college curriculum. They are a recent importation. Their exact position and relations are scarcely yet settled, and one may easily fall into the mistake, on the one hand, of unwarranted precision in setting forth their present status, and, on the other, of overconfidence in predicting their ultimate influence upon the culture of our higher institutions of learning. Our observation, however, has probably extended over a period sufficiently long to yield some reliable results, which at this stage of it may well be brought together.

I. What were the circumstances under which the sciences gained a place in our educational machinery?

The college curriculum in its present form is the result of a gradual growth from very ancient and rude beginnings. As in a living organism, the successive modifications of the bulk and complexity of its structure have been closely dependent upon its environment. It responds with great sensitiveness to changes in the world about it. Hence it comes to pass that the apparatus and methods of culture of one period and race differ more or less widely from those of all other periods and races. The history of this development is inextricably intertwined with the progress of external events. We must look, therefore, without, if we would find the explanation of the last great modification of the means of education.

Of course, science in some form and to some extent had a place in education long before the period which $\mathrm{I}$ now have in mind. On the other hand, in some quarters it may be said to be still fighting for recognition even at the present moment. Moreorer, periods glide insensibly into succeeding periods. There are no sharp lines in nature. For that reason there can be none in history. And yet, in order to avoid confusion and irksome modifications of every statement, I must be allowed to draw a somew hat arbitrary line and consciously to foreshorten the stages of a continuous advance.

For reasons which seem to me sufficient, I draw the line at 1859 , the date of the publication of the "Origin of Species," and characterize the 35 years following as the period of science in education. It will, perhaps be agreed that no book in the domain of science. not even excepting the work of Bacon or Newton, has produced an influence so far-reaching and so profound. This date I fix upon the more willingly, inasmuch as it marks the new birth of the science of biology, which has affected all departments of human thought more deeply and permanently than all the other sciences. And it I have chiefly in mind on the present occasion.

The characteristic feature of the intellectual life of the period since the publication of the "Origin of Species" is the ferment precipitated by its doctrine. And the education of the period, in its spontaneous adjustment to external conditions, wears the same unsettled complexion, with science for its dominant tone. The middle decades of this century are unrivalled in all the thrilling history of the development of natural knowledge. The "Report on the Progress of Science" during the twenty years next following the Revolution of 1789 and read before the Emperor Napoleon in 1808, while it records some great names, contains nothing to match the record of the fcrties and fifties. And the next thirty years carried the wave of discovery and generalization but little higher. But about 1840 the spirit of scientific inquiry grew more intense, laid under contribution a larger number of rarely equipped minds, and pressed forward to attack the problems of the physical universe with a degree of vigor, boldness, and consecration which could not fail of brilliant achievements. Since that epoch the application of machinery to industrial production and to locomotion and intercommunication has revolutionized our common lives and given us new standards of comfort and activity. This revolution in the external aspects of modern civilization, it must be observed, "has been preceded, accompanied,

1 Abstract of a paper read before the North Carolina College Association a Raleigh, Feb. 25, 1893 . 